\title{
Der Europaausschuss des dänischen Folketing. Eine Analyse der Verhandlungsmandate
}

\author{
Tim Mickler
}

Die Europäische Union ist aus der Politik des 21. Jahrhunderts nicht mehr wegzudenken. Ausgehend von der Grundidee, europäische Staaten durch gezielte wirtschaftliche Verflechtung in gegenseitige Abhängigkeit zu bringen, wurde mit dem Inkrafttreten des Vertrags über die Gründung der Europäischen Gemeinschaft für Kohle und Stahl (EGKS) am 23. Juni 1952 der Grundstein für die europäische Einigung gelegt. Durch mehrere Reformverträge wurden die supranationalen Kompetenzen ausgebaut, und die Institutionen der im Jahr 2011 aus 27 Mitgliedstaaten bestehenden Europäischen Union haben weitreichende Kompetenzen, das Leben der rund 500 Millionen Einwohner zu regeln.

Dies bringt die Frage nach dem Verbleib der nationalen Parlamente als direkt gewählte Interessenvertretungen der Bevölkerungen mit sich. Diese naturgemäß auf den Nationalstaat begrenzten Institutionen werden im europäischen Einigungsprozess als Verlierer angesehen. ${ }^{1}$ Besonders die Ausübung der Kontrollfunktion gegenüber der eigenen Regierung in Angelegenheiten der EU scheint durch Informationsasymmetrien gefährdet. Parlamente als „erstaunlich anpassungsfähige Organisationen, die durch einen beständigen Wandlungsprozess ihre Leistungsfähigkeit bewahren konnten "2, haben auf diese Entwicklung reagiert und durch die Institutionalisierung von Informations- und Mitbestimmungsrechten gegenüber ihren Regierungen ihren Einfluss in Angelegenheiten der Europäischen Union zu bewahren versucht. Diese Entwicklung hat zu einer Vielfalt von Strukturen und Verfahren geführt, wobei dem dänischen Folketing und seinem Europaausschuss in der Forschung besondere Aufmerksamkeit gewidmet wurde. Seinen Ursprung hat der Ausschuss bereits 1961, also zwölf Jahre vor Einritt Dänemarks in die EU³; er hat somit als Vorreiter andere Parlamente beeinflusst. Vergleichende Studien ${ }^{4}$ zeigen eine beachtliche Übereinstimmung:

1 Vgl. Tapio Raunio, Always One Step Behind? National Legislatures and the European Union, in: Government and Opposition, 34. Jg. (1999), H. 2, S. 180 - 202; Andreas Maurer, Parlamentarische Demokratie in der Europäischen Union - Der Beitrag des Europäischen Parlaments und der nationalen Parlamente, Baden-Baden 2002; Katrin Auel / Arthur Benz, The Europeanisation of the German Bundestag: Institutional Change and Informal Adaptation, in: German Politics, 15. Jg. (2006), H. 3, S. $249-268$.

2 Gerhard Loewenberg, The Role of Parliaments in Modern Political Systems, in: ders. (Hrsg.), Modern Parliaments: Change or Decline?, Chicago / New York 1971.

3 Dieses Gremium mit dem Namen „Folketingsausschuss zur Begleitung der Regierungsverhandlungen über Dänemarks Aufnahme in die Europäische Wirtschaftsgemeinschaft" (Folketingets udvalg til at følge regeringens forhandlinger om Danmarks optagelse I det europæiske økonomiske Fælleskab) hatte die Aufgabe, den Gesetzentwurf über den Beitritt Dänemarks in die EWG zu erarbeiten. Aufgrund der Länge des Namens bürgerte sich in kurzer Zeit der Name „Marktverhandlungsausschuss" oder "Marktausschuss" (Markedudvalg) ein.

4 Vgl. Torbjörn Bergmann / Wolfgang C. Müller / Kaare Strøm, Introduction: Parliamentary Democracy and the Chain of Delegation, in: European Journal of Political Research, 37. Jg. (2000), H. 3, S. 255 - 260; Andreas Maurer / Wolfgang Wessels (Hrsg.), National Parliaments on Their Ways to Europe: Losers or Latecomers?, Baden-Baden 2001; Olivier Rozenberg, The Involvement of National Parliaments in European Union Affairs. An Empirical Test of Two Variables, Paper for 
Diese Arbeiten klassifizieren die nationalen Parlamente aufgrund Ihrer Fähigkeit, effektive Kontrolle auszuüben als starke oder schwache Kontrolleure. Das dänische Parlament nimmt in all diesen Arbeiten eine Spitzenposition ein; sein Europaausschuss wird immer wieder als der stärkste seiner Art bezeichnet. Während sich frühere Arbeiten primär auf die formalen Kompetenzen konzentriert, soll hier analysiert werden, wie das dänische Modell in der politischen Praxis funktioniert.

\section{Das dänische Modell}

Das dänische Modell besteht aus einem zentralen Europaausschuss (Europaudvalg, EUA) mit weitreichenden Informationsrechten gegenüber der Regierung und der Möglichkeit, bindende Verhandlungsmandate zu erteilen. Er ist der Mittelpunkt in der Bearbeitung von Angelegenheiten der EU im parlamentarischen System. Die Hauptaufgabe besteht in der Kontrolle der Europapolitik der Regierung; seine Kompetenzen sind jedoch seit seiner Institutionalisierung stetig gewachsen, und er ist verantwortlich für die Koordinierung der Bearbeitung von Angelegenheiten der EU im Folketing. Der EUA bildet die Arena, in der die Auseinandersetzung von Repräsentanten der Fraktionen beziehungsweise Parteien strukturiert wird. ${ }^{5}$ Wie fast alle anderen Ständigen Ausschüsse des Dänischen Parlaments besteht er aus 17 Mitgliedern und zehn bis zwölf Stellvertretern, ist jedoch traditionell einer der prestigeträchtigsten und begehrtesten Ausschüsse. Nach einer Studie von Henrik Jensen ist er zusammen mit dem Finanzausschuss das Gremium, dessen Mitglieder in überdurchschnittlichem Maße hochrangige Positionen in ihren Parteien (Vorsitzende, stellvertretende Vorsitzende, politische Wortführer) bekleiden. ${ }^{6}$

Während sich in dem Gesetz über den Beitritt zur EG (tiltrædelsesloven) und in der Geschäftsordnung des Folketing nur sehr grundlegende Regelungen finden, besteht für die Ausschussmitglieder die Möglichkeit, durch Verfahrensberichte (beretninger) bindende Vereinbarungen mit der Regierung ${ }^{7}$ zu verabschieden und die Zusammenarbeit nach ihren Vorstellungen zu präzisieren.

So können die Fraktionen im Ausschuss den Großteil ihrer Kompetenzen selber regeln und relativ kurzfristig auf Entwicklungen auf europäischer Ebene reagieren. Insgesamt 28 Verfahrensberichte hat der Europaausschuss seit seinem ersten Bericht am 29. März 1973 bis ins Jahr 2010 verabschiedet. Dies hat zu einem Verfahren mit höchst standardisierter und extensiver Informationspflicht der Regierung geführt, an dessen Ende die Regierung den Ausschuss in einer Sitzung über Angelegenheiten von wesentlicher Bedeutung (væsentlig betydning) in Kenntnis setzt und bei solchen von großer Reichweite (større rækkevider)

the ECPR Joint Sessions of Workshops, Turin 2002; Tapio Raunio, Holding Governments Accountable in European Affairs: Explaining Cross-National Variation, in: The Journal of Legislative Studies, 11. Jg. (2005), H. 3/4, S. $319-342$.

5 Vgl. Erik Damgaard / Asbjørn Sonne Nørgaard, The European Union and Danish Parliamentary Democracy, in: The Journal of Legislative Studies, 6. Jg. (2000), H. 1, S. 33 - 58, S. 41.

6 Vgl. Henrik Jensen, Europaudvalget. Et udvalg i Folketinget, Aarhus 2003.

7 Vgl. Carsten Schymik, Der Europaausschuss des dänischen Folketing - der machtvollste Europas?, Friedrich-Ebert-Stiftung, Internationale Politikanalyse, Berlin 2008, S. 10, http://library.fes.de/ pdf-files/id/ipa/05640.pdf (Abruf am 27. März 2010). 
vor einer Abstimmung auf europäischer Ebene um ein Verhandlungsmandat ersucht. ${ }^{8}$ Dabei präsentiert die Regierung zu einer bestimmten Sache ihr beabsichtigtes Verhalten und lässt es sich vom Ausschuss „absegnen“. In der beschlussfassenden Sitzung legt der zuständige Fachminister mündlich dar, mit welcher Position die Regierung beziehungsweise der Minister in die Verhandlungen im Rat zu gehen beabsichtigen.

Vor der Präsentation des Verhandlungsmandats wird die Angelegenheit inhaltlich skizziert, mögliche Konsequenzen für Dänemark erörtert, Kritik von Seiten der Regierung und Interessengruppen vorgetragen. Durch das umfassende Berichtswesen sind die Mitglieder des Ausschusses zwar schon vorher über den Inhalt informiert, jedoch „kennen [sie] in der Regel nicht die spezifischen Positionen der Verhandlungsgrundlage, die sie mandatieren sollen"?.

Im Anschluss an die Vorlage der Regierungsposition haben die Ausschussmitglieder die Gelegenheit, Nachfragen zu stellen. Die EU-Sprecher der Fraktionen spielen hier eine zentrale Rolle, da sie an diesem Punkt auch eine Stellungnahme für oder gegen die vorgeschlagene Position abgeben. Als Gegenstimme wird nur eine ausdrückliche Ablehnung gewertet; kritische Anmerkungen, keine Äußerungen und Nicht-Anwesenheit eines Abgeordneten einer Fraktion, sofern diese einen Sitz im Ausschuss hat, werden nicht als Ablehnung gewertet. Es ist jedoch möglich, bei Nicht-Anwesenheit den Vorsitzenden im Vorfeld einer Ablehnung bezüglich eines bestimmten Mandats zu informieren.

Der entscheidende Moment der Mandatierung liegt am Ende der Sitzung, wenn der Ausschussvorsitzende feststellen kann, ob eine Mehrheit gegen die Verhandlungsposition der Regierung gestimmt hat oder nicht. Bei Ausbleiben einer solchen Mehrheit gilt das Mandat als erteilt. Dieses System folgt der typischen Logik des „negativen Parlamentarismus": Die Regierung muss keine Mehrheit finden, sondern lediglich sicherstellen, dass es keine Mehrheit gegen ihre Position gibt. ${ }^{10}$ Die Sprecher werden dabei als Repräsentanten ihrer Fraktion angesehen, so dass angenommen wird, dass alle ihre Mitglieder im Plenum ebenfalls so abstimmen würden. ${ }^{11}$

Eine Ablehnung der Position bedeutet, dass der Minister ein neues Mandat vorlegen muss. Er kann das bisherige auch komplett zurückziehen und ein neues Mandat in einer späteren Sitzung des Europaausschusses präsentieren. Dies hängt vom Zeitplan der europäischen Institution ab, da das Verhandlungsmandat in jedem Fall vor einer Abstimmung im Ministerrat präsentiert werden muss.

8 Die grundlegende Einschätzung, ob eine Angelegenheit "große“ oder „wesentliche“ Bedeutung hat, liegt im Ermessen der Regierung. Der Europaausschuss muss diese Einschätzung jedoch nicht hinnehmen, sondern kann den Minister auch dazu bringen, in einer Angelegenheit die Position der Regierung vorzustellen, obwohl der Europaausschuss über diese Angelegenheit ursprünglich nur informiert werden sollte.

9 Carsten Schymik, a.a.O. (Fn. 7), S. 12.

10 Vgl. Arthur Benz, Europäisierung der Arbeit nationaler Parlamente, Abschlussbericht, Hagen 2004, S. 10, http://www.fernunihagen.de/imperia/md/content/politikwissenschaft/lgi/dfgii_abschlussbericht.pdf (Abruf am 27. März 2010).

11 Vgl. Folketingets EU-Oplysning, Consideration of EU Matters in the Folketing - A Summary, Kopenhagen 2007, Annex 2: European Affairs Committee Voting Rules, S. 23, http://eu-oplysningen.dk/upload/application/pdf/be607a32/EU_matters_in_the\%20_Folketing.pdf (Abruf am 27. März 2010). 
Ob dieses System - formal betrachtet machtvoll - auch in der politischen Realität seine beabsichtigte Wirkung entfalten kann, ist bisher unzureichend erforscht. Der Europaausschuss räumt ein, dass eine Ablehnung des Verhandlungsmandats „vergleichsweise selten “12 ist. Carsten Schymik endet in seinem sehr guten Überblick an diesem Punkt: Wie der Europaausschuss zu einer Entscheidung komme, ob und wie er Einfluss auf das Verhandlungsmandat im Vorfeld nimmt, „müssten weitere Forschungen erweisen“"13.

\section{Theoretische Grundlage: der Prinzipal-Agenten-Ansatz}

Mithilfe der Prinzipal-Agenten-Theorie (kurz: Agententheorie) lässt sich das Verhältnis von Regierung und Parlament in parlamentarischen Regierungssystemen theoretisch fassen, da sie Situationen beschreibt, in denen ein Agent, die Regierung, im Auftrag eines Prinzipals, das Parlament, handelt. Ursprünglich entwickelt, um Informationsasymmetrien zwischen Vertragspartnern in ökonomischen Beziehungen darzustellen, wurde erstmals durch $D$. Roderick Kiewit und Mathew McCubbins auf politische Repräsentation erweitert, indem der Fokus weniger auf den Vertrag als auf Autorität gelegt wird. Nach ihrer Definition besteht eine Agentenbeziehung, wenn ,an agent is delegated (...) the authority to take action on behalf of $(. .$.$) the principal "14. Delegation ist definiert als das Übertragen von Entschei-$ dungsbefugnis von denen, die diese innehaben, an andere, die in ihrem Namen dann eine Entscheidung treffen. ${ }^{15}$

Gleichzeitig ist der Agent dem Prinzipal verantwortlich, da Ersterer verpflichtet ist, an Stelle des Prinzipals zu handeln und der Prinzipal wiederum befähigt ist, den Agenten aufgrund seines Verhaltens zu belohnen oder zu bestrafen. ${ }^{16}$ Grundlegend wird angenommen, dass in Prinzipal-Agenten-Beziehungen einerseits Informationsasymmetrien zugunsten des Agenten und zweitens Interessenkonflikte zwischen den Parteien bestehen ${ }^{17}$, und jede Delegation von Autorität die Gefahr mit sich bringt, dass der Agent nicht im Sinne des Prinzipals handelt. Dies wird als Agenturverlust (agency loss) bezeichnet.

Der Prinzipal ist daran interessiert, die Wahrscheinlichkeit solcher Agenturverluste möglichst zu minimieren. Im Idealfall wird er den Agenten sorgfältig auswählen, ihn überwachen, um Fehlverhalten zu entdecken und darüber hinaus noch in der Lage sein, dem Agenten mit wirkungsvollen Sanktionen zu drohen.

12 Folketingets EU-Oplysning, The Folketing's European Affairs Committee, Kopenhagen 2006, S. 8, http://eu-oplysningen.dk/fakta_en/pub/euu (Abruf am 18. April 2010).

13 Carsten Schymik, a.a.O. (Fn. 7), S. 8.

14 D. Roderick Kiewiet / Mathew D. McCubbins, The Logic of Delegation: Congressional Parties and the Appropriations Process, Chicago 1991, S. 240. Vgl. auch Thomas Saalfeld, Members of Parliament and Governments in Western Europe: Agency Relations and Problems of Oversight., in: European Journal of Political Research, 37. Jg. (2000), H. 3, S. 353 - 376, S. 355.

15 Vgl. Kaare Strøm, Delegation and Accountability in Parliamentary Democracies, in: European Journal of Political Research, 37. Jg. (2000), H. 3, S. 261 - 289, S. 266.

16 Vgl. ebenda, S. 267.

17 Vgl. Philipp Harfst / Kai Uwe Schnapp, Instrumente parlamentarischer Kontrolle der Exekutive in westlichen Demokratien, Wissenschaftszentrum Berlin für Sozialforschung (WZB), Veröffentlichungsreihe der Abteilung Demokratie: Strukturen, Leistungsprofil und Herausforderungen des Forschungsschwerpunkts Zivilgesellschaft, Konflikte und Demokratie, Berlin 2003, S. 5, http://www.uni-lueneburg.de/fb2/soz/politik/seiten/team/dokumente/IV03-201.pdf (Abruf am 16. April 2010). 
Innerhalb dieses Modells gibt es vier Mechanismen, mit denen der Prinzipal auf Agenturverluste reagieren kann, die weiter in ex-ante Mechanismen (Zeitpunkt vor dem vertraglichen Beginn der Prinzipal-Agenten-Beziehung) und ex-post Mechanismen (Zeitpunkt nach Einsetzung des Agenten durch den Prinzipal) unterschieden werden.

(1) Durch Prüf- und Auswahlmechanismen kann der Prinzipal verhindern, dass er den falschen Agenten beauftragt (screening and selection mechanism), und dafür sorgen, dass er jenen bekommt, dessen Ziele und Präferenzen seinen entsprechen. ${ }^{18}$

(2) Ebenso vor Vertragsabschluss kann der Prinzipal durch die Art der Vertragsgestaltung (contract design) die Beziehung zum Agenten beeinflussen und durch Anreize dessen Verhalten an seine Präferenzen binden.

(3) Durch Beobachtung und die Anforderung von Berichten (monitoring and reporting requirements) wird der Prinzipal nach Vertragsabschluss befähigt, eine ausreichende Kontrolle auszuüben.

(4) Durch institutionelle Begrenzungen (institutional checks) soll der Agent zur Zusammenarbeit verpflichtet werden. Shawn Reichert und Bernadette Jungblut sprechen in diesem Zusammenhang von Sanktionen, um deutlich zu machen, dass die Anreizstruktur, geschaffen durch die Vertragsgestaltung, erst durch die Androhung von Sanktionen wirksam wird. ${ }^{19}$

Im Falle einer großen Sorge vor adverse selection wird der Prinzipal daran interessiert sein, die ex-ante Mechanismen stärker auszuprägen. Bei stärkerer Besorgnis über moral hazard-Verhalten liegt der Fokus auf den ex-post Mechanismen.

Die Informationsasymmetrie zugunsten der Regierung in Verbindung mit der Gesetzgebung auf europäischer Ebene kann zu Output-Ergebnissen gegen den Willen des Parlaments führen. Um die Differenz zwischen dem gewünschten und dem tatsächlichen Ergebnis zu minimieren, sind die Verhandlungsmandate ein Mittel, um im Vorfeld die Interessen beider Seiten abzustimmen. Sie stellen somit eine extreme Form der „monitoring and reporting requirements" dar. Die Regierung wird kontinuierlich und detailliert überwacht und muss sicherstellen, dass sie eine Mehrheit des Parlaments hinter sich hat. Dabei handelt es sich um eine so genannte win-win-Situation. Die Mandatierung ihrer Verhandlungsposition gibt der Regierung einen gewissen Grad an Sicherheit, dass sie sich nach einer Abstimmung auf europäischer Ebene nicht mit einem Misstrauensvotum konfrontiert sieht, sofern sie bei diesem Mandat bleibt; das Parlament wird durch die Mandatierung über das Verhalten der Regierung in supranationalen Verhandlungen informiert und begrenzt die Wahrscheinlichkeit ungewollter Ergebnisse. In diesem Sinne bleibt zu klären, ob die Fraktionen im Ausschuss durch dieses System auch faktisch Einfluss auf die Regierung ausüben können, oder ob es sich beim Europaausschuss um einen zahnlosen Tiger handelt. Dazu muss untersucht werden, wie oft sich eine Mehrheit gegen die Position der Regierung findet und wie die Mitglieder des Ausschusses probieren, ihren Einfluss geltend zu machen.

18 Vgl. M. Shawn Reichert / Bernadette M. E Jungblut, European Union External Trade Policy: Multilevel Principal-Agent Relationships, in: The Policy Studies Journal, 35. Jg. (2007), H. 3, S. 395 -418 , S. 398.

19 Vgl. ebenda. 


\section{Methodisches Vorgehen}

Um mit realen Zahlen ${ }^{20}$ arbeiten zu können, wurden in einem ersten Schritt die Ergebnisse der Abstimmungen über die Verhandlungsmandate in den Beschlussprotokollen (beslutningsreferater) im Zeitraum von 2000 bis Ende 2009 analysiert ( $\mathrm{n}=456$ Beschlussprotokolle mit 1.029 Verhandlungsmandaten). Diese geben an, welche Fraktionen sich gegen das Verhandlungsmandat ausgesprochen haben. Deren Sitze wurden addiert und auf eine mögliche Mehrheit überprüft.

Betrachtet man die Anzahl der angenommenen und abgelehnten Mandate, ohne den Inhalt der Position der Regierung einzubeziehen, besteht die Gefahr der Fehlinterpretation. Eine kritische Position des Ministers würde von EU-skeptischen Parteien eher akzeptiert werden als eine Unterstützung des Vorhabens auf europäischer Ebene. Hierzu muss auf die Sitzungsprotokolle zurückgegriffen werden. ${ }^{21}$ Auch hier wurden alle verfügbaren Dokumente bis zur letzten Sitzung des Jahres 2009 in die Analyse einbezogen und die Art des Verhandlungsmandats ${ }^{22}$ in eine von drei Kategorien eingeordnet: Unterstützung, Ablehnung oder Differenziertes Verhandlungsmandat, also ein Mandat mit Optionen bei bestimmten Entwicklungen im Rat. Es wird gezeigt werden, dass der Minister in fast allen Fällen keine Mehrheit gegen seine Position vorfindet und sich die Regierung positiv gegenüber einem Großteil der Gesetzgebung auf europäischer Ebene zu verhalten beabsichtigt. Wie bereits erwähnt, müsste die Regierung, verfügt sie über eine Mehrheit im Parlament, lediglich ihre eigene(n) Fraktion(en) mobilisieren, um ihr Verhandlungsmandat bestätigt zu bekommen. Nun ist der dänische Fall insofern speziell, als es in der Zeitspanne seit dem EU-Beitritt Dänemarks im Jahr 1973 nur für 20 Monate eine Mehrheitsregierung gab ${ }^{23}$ : „Denmark holds the postwar world record with regard to minority government rule in a parliamentary government." ${ }^{24}$ Ausgehend von den Minderheitsregierungen in allen Wahlperioden des Untersuchungszeitraums und der logischen Konsequenz, dass keine Koalition nur durch ihre eigenen Stimmen das Verhandlungsmandat ausstellen kann, ist also die Frage zu klären: Wer sorgte für die Mehrheit? Folgende Erklärungsmuster wurden dazu überprüft:

(1) Das regierende Wahlbündnis (valgforbund), bestehend aus Regierungsparteien und Stützparteien, trägt die Position des Ministers und sorgt für die nötige Mehrheit.

(2) Der Minister ändert sein Verhandlungsmandat während der Sitzung und verhindert so eine Ablehnung; die Verhandlungsmandate werden ausgiebig diskutiert, bevor es zu einer Abstimmung kommt.

20 Das Folketing führt keine Statistik über die Anzahl der abgelehnten und angenommenen Verhandlungsmandate.

21 Diese sind seit Oktober 2006 frei zugänglich und enthalten Mitschriften der Diskussionen von allen Punkten, die nicht vertraulich behandelt wurden.

22 Das Verhandlungsmandat wird in den Protokollen durch das Kürzel FO (forhandlingsoplæg) gekennzeichnet.

23 Bestehend aus einer Koalition von Socialdemokraterne, Det Radikale Venstre, Centrum-Demokraterne und den Kristendemokraterne unter dem Sozialdemokraten Poul Nyrup Rasmussen.

24 Erik Damgaard, Denmark: Delegation and Accountability in Minority Situations, in: Kaare Strøm / Wolfgang C Müller / Torbjörn Bergman (Hrsg.), Delegation and Accountability in Parliamentary Democracies, Oxford 2008, S. 281 - 300, S. 282. 
(3) Im Vorfeld der beschlussfassenden Sitzung wurden die Positionen abgestimmt, und der Minister ist über die „Stimmungslage“ im Ausschuss informiert.

Um Erklärungsmuster 1 zu überprüfen, müssen die Zugehörigkeit zu einem Wahlbündnis und der Grad an Euroskeptizismus ${ }^{25}$ auf ihre Erklärungskraft gegenüber dem tatsächlichen Abstimmungsverhalten berechnet werden. Die Zusammensetzung der Wahlbündnisse in den einzelnen Wahlperioden wurde aus der Auflistung in den Jahrbüchern des Folketing (Folketingtidende) ${ }^{26}$ ermittelt. Die Gruppierung der Fraktionen nach ihrem Grad an Euroskeptizismus orientiert sich an den Kriterien des von Aleks Szczerbiak und Paul Taggart vorgeschlagenen Modells von hartem und weichem Euroskeptizismus. Ersterer beschreibt Parteien, die die europäische Integration prinzipiell ablehnen, einen Austritt aus der EU befürworten oder eine policy vertreten, die der Europäischen Union grundsätzlich entgegengesetzt ist. Unter weichen Euroskeptizismus fallen nach dieser Klassifikation Parteien, die keine grundsätzlichen Einwände gegen eine Mitgliedschaft haben, sondern deren Bedenken sich gegen einzelne policy-Bereiche richten, oder die die EU im Widerspruch zu nationalen Interessen sehen. ${ }^{27}$ Die Klassifizierung hier basiert auf den Eigendarstellungen der Parteien auf ihren Websites, dem Internetauftritt des Folketing und, soweit verfügbar, bereits durchgeführter Forschung. Es wurden alle Fraktionen klassifiziert, die im Untersuchungszeitraum einen Sitz im Europaausschuss hatten. ${ }^{28}$

Die Ergebnisse dieses Teils der Analyse wurden in den Datensatz mit dem Abstimmungsverhalten der Fraktionen eingefügt. ${ }^{29}$ Für die Variablen „Grad des Euroskeptizismus“30 und „Wahlbündnis“ wurde anschließend die Stärke des Zusammenhangs mit der Variable „Abstimmungsverhalten“ mithilfe des Pearsonschen Korrelationskoeffizienten errechnet.

25 Der Analyse liegt die Annahme zu Grunde, dass Gesetzgebung auf europäischer Ebene immer ein "Mehr" an politischer Integration in Richtung EU bedeutet. Politische Integration ist nach Haas definiert als ,the process whereby political actors in several distinct national settings are persuaded to shift their loyalties, expectations and political activities toward a new centre, whose institutions possess or demand jurisdiction over the pre-existing national states. The end result of a process of political integration is a new political community, superimposed over the pre-existing ones" (Ernst B. Haas, The Uniting of Europe. Political, Social and Economic Forces 1950-1957, Stanford 1968, S. 16). Es erscheint naheliegend, dass sich euroskeptische Parteien eher zurückhaltend gegenüber europäischer Gesetzgebung verhalten, da diese eine fortschreitende Integration mit sich bringt.

26 Das Folketingtidende enthält eine Übersicht über die Arbeit des letzten Kalenderjahres und ist leider nicht elektronisch zugängig. Die erforderlichen Seiten wurden per E-Mail von Rikke Friis Touborg am 20. Mai 2010 bereitgestellt (folketinget@ft.dk).

27 Vgl. Aleks Szczerbiak / Paul Taggart, The Party Politics of Euroscepticism in EU Member and Candidate States, Sussex European Institute, SEI Working Paper No 51, Brighton 2003, http:// www.sussex.ac.uk/sei/documents/epern-working-paper-6.pdf (Abruf am 3. Mai 2010).

28 Sowohl die Liberal Alliance als auch die Fremskridtsparti waren zwar im Folketing vertreten, hatten jedoch keine Sitze im Ausschuss bekommen und spielen somit in dieser Analyse keine Rolle. Die Parteien der Färöer-Inseln und Grönlands haben keinen festen Platz im Europaausschuss.

29800 Daten stammen aus einer sozialdemokratisch-sozialliberalen (Socialdemokraterne - Det Radikale Venstre) und 1.032 Daten aus einer rechtsliberal-konservativen Regierungskoalition (Venstre - Det Konservative Folkeparti).

30 Da es sich bei der dritten Variable um eine nominalskalierte handelt, wurde diese dichotomisiert (Nicht-Euroskeptisch - Euroskeptisch), um den Korrelationskoeffizienten nach Pearson ausrechnen zu können. 
Für Erklärungsmuster 2 wurden die Protokolle hinsichtlich Änderungen des Verhandlungsmandats und der Anzahl der Nachfragen der Fraktionen analysiert:

(1) Eine Änderung der Position des Ministers muss in den Protokollen durch das Kürzel Æ (ændring - Änderung) deutlich gemacht werden. Die Anzahl dieser Änderungen wurde in allen 279 vorliegenden Protokollen bis zur letzten Sitzung des Jahres 2009 ermittelt.

(2) Zur Messung, welche Fraktionen sich während der Beratungen eines Verhandlungsmandats öfter äußern und mehr Nachfragen stellen, wurde die Anzahl der Beiträge von allen Abgeordneten innerhalb der Diskussionen ermittelt und den jeweiligen Fraktionen zugeordnet. ${ }^{31}$ Damit soll die Frage geklärt werden, welche von ihnen sich aktiver und welche sich passiver verhalten und Rückschlüsse über die Intensität der Beratungen gezogen werden. Ermittelt wurden erstens die Anzahl der Nachfragen einer Fraktion und die Anzahl der Nachfragerunden insgesamt.

Ebenso ist nicht nur eine Änderung während der Ausschusssitzung denkbar, sondern auch die Beeinflussung oder Abstimmung der Regierungsposition im Vorfeld (Erklärungsmuster 3). Hier konnte jedoch nicht auf die Protokolle zurückgegriffen werden ${ }^{32}$; daher wurden leitfadengestützte Experteninterviews mit Benny Engelbrecht (Socialdemokraterne), Pia Adelsteen (Dansk Folkeparti) und einem Mitarbeiter des EU-Sekretariats, der um Anonymität gebeten hat, im Mai 2010 in Kopenhagen durchgeführt. Der Fokus dieser Interviews lag auf der Willensbildung innerhalb der Parteien und der Behandlung der Gesetzesvorschläge, für die später ein Verhandlungsmandat präsentiert wird, in der Zeit vor der beschlussfassenden Sitzung.

\section{Ergebnisse: Überprüfung der Erklärungsmuster}

Aufgrund der Ergebnisse kann man nur konstatieren, dass die eigene Darstellung des Europaausschusses, dass die Position der Regierung „vergleichsweise selten“33 abgelehnt wurde, fast schon untertrieben anmutet.

Von 1.029 analysierten Verhandlungsmandaten findet sich bei 999 keine Mehrheit gegen die Position der Regierung (97,1 Prozent). In nur 20 Fällen stimmte eine Mehrheit des Europaausschusses gegen das Verhandlungsmandat (1,9 Prozent).

Die eigentliche Position der Regierung kann nur durch inhaltliche Analyse der Sitzungsprotokolle ermittelt werden, die seit Oktober 2006 zugänglich sind ( $\mathrm{n}=279)$. In 196 Fällen (70,3 Prozent) lief das Verhandlungsmandat des Ministers auf eine Unterstützung des Vorschlags im Ministerrat hinaus. Eine ablehnende Haltung wurde hingegen nur neunmal (3,2 Prozent) präsentiert, während in 50 Fällen (17,9 Prozent) ein Differenziertes Verhandlungsmandat vorgelegt wurde. ${ }^{34}$

31 Es konnten nur die Fälle einbezogen werden, in denen weder ein Verhandlungsmandat zurückgezogen wurde noch der Inhalt der Diskussion vertraulich war $(\mathrm{n}=255)$.

32 Einerseits konnte nicht auf die Protokolle zurückgegriffen werden, weil es unmöglich ist, die komplette Bearbeitung eines bestimmten Gesetzesvorschlags in ihnen nachzuvollziehen und andererseits da die meisten Behandlungen im Vorfeld vertraulich stattfinden (lukkede døre, beziehungsweise lukket) und somit keine vollständige Analyse möglich wäre, selbst wenn man die Bearbeitung in den Agenden nachvollziehen könnte.

33 Folketingets EU-Oplysning, a.a.O. (Fn. 12), S. 8.

34 In 16 Fällen (5,7 Prozent) war der Inhalt vertraulich, in acht Fällen (2,9 Prozent) stand kein Verhandlungsmandat zur Debatte. 


\begin{tabular}{|l|c|c|c|}
\hline Tabelle 1: Verhandlungsmandate im Europaausschuss des Folketing (Oktober 2000 bis Dezember \\
2009)
\end{tabular}

Als Ausgangspunkt für die Analyse kann man somit festhalten, dass der Europaausschuss in der letzten Phase der Behandlung eines Gesetzesvorschlags vor dessen Beratung im Ministerrat sehr selten interveniert hat. Die Position des Ministers war in einem Großteil der Fälle auf Unterstützung und in den wenigsten Fällen auf Ablehnung im Ministerrat gerichtet; in knapp 18 Prozent der Fälle präsentierte die Regierung ein differenziertes Verhandlungsmandat.

\subsection{Euroskeptizismus und Abstimmungsverhalten}

Einheitlich abstimmende Wahlbündnisse, die über eine parlamentarische Mehrheit verfügen, können die Regierung stützen; es ist jedoch auch denkbar, dass die Abgeordneten ihr Abstimmungsverhalten an ihrem Grad des Euroskeptizismus orientieren.

Euroskeptizismus ist eine Erscheinungsform des politischen Widerstands im europäischen Integrationsprozess. Die Dänen haben sich seit ihrem EU-Beitritt 1973 durch eine bemerkenswerte Zurückhaltung gegenüber einer weiteren Integration ausgezeichnet, die in der Ablehnung des Maastrichter Vertrags gipfelte und zu den dänischen Opt-outs bei der Währungsunion, der Verteidigung und in der Zusammenarbeit im Bereich Justiz und Inneres führte.

Zwar lässt sich ein Prozess beobachten, in dem die Mitgliedschaft in der EU prinzipiell mehr und mehr als etwas Gutes gesehen wird, aber viele Dänen stehen einer fortschreitenden Integration kritisch gegenüber und zeigen - wie die schwedische Bevölkerung - zudem „strong electoral support for Eurosceptical parties“ 35 .

Die Analyse der Parteien, die seit dem Jahr 2000 einen Sitz im Europaausschuss hatten, nach den Kriterien für parteibasierten Euroskeptizismus von Taggart und Sczerbiak ergab folgendes Bild: Die Parteien Socialdemokraterne, Venstre, Radikale Venstre und CentrumDemokraterne sind positiv gegenüber der EU eingestellt. Während sich die SD für eine volle Mitgliedschaft ohne Ausnahmeregelungen ${ }^{36}$ ausspricht und die pro-europäische Venstre auf ihrer Internetseite erklärt, dass sie die Ausnahmeregelungen gern erneut zur

35 Tapio Raunio, Softening but Persistent: Euroscepticism in the Nordic EU Countries, in: Acta Politica, 42. Jg. (2007), H. 2, S. 191 - 210, S. 192.

36 Vgl. Folketing, Socialdemokraterne. Danmark og EU, 2009, http://www.ft.dk/Demokrati/Partier/partiernesPolitik/detMenerPartierneOm/EU/Danmark\%20og\%20EU.aspx?party=A (Abruf am 22. September 2011). 
Debatte stellen möchte ${ }^{37}$, spricht sich die Radikale Venstre für eine Vollmitgliedschaft ohne Vorbehalte - „je eher, desto besser“38 - aus. Auch die Centrum-Demokraterne äußern sich positiv zu einer fortschreitenden Integration. 39

Die Socialistisk Folkeparti ist in Fragen der europäischen Einigung tief gespalten; Mitgliedschaft in der Europäischen Währungsunion wird einerseits als „Katastrophe für die dänische Ökonomie “ 40 angesehen, und man ist gegen Vollmitgliedschaft, andererseits aber für eine Zusammenarbeit in bestimmten Feldern. Det Konservative Folkeparti betont die Wichtigkeit eines geeinten Europas für Dänemark, sieht jedoch als konservative Partei den Nationalstaat als Ausgangspunkt für „Kultur, Sprache und den Rahmen für das dänische Leben “ 41 an; Entscheidungen sollen so nah wie möglich getroffen werden. Vergleichbar urteilen die Kristendemokraterne, da sie die Wichtigkeit des Subsidiaritätsprinzips und den Verbleib bestimmter Aufgaben bei den Ländern betonen und der Europäischen Union nur eine unterstützende Rolle einräumen wollen. ${ }^{42}$

Harte Euroskeptiker sind die Dansk Folkeparti und die Enhedslisten-De Rød-Grønne. Erstere „bekämpft jeden Versuch, einen europäischen Verbundstaat zu schaffen“ ${ }^{43}$, setzt sich für ein stärkeres Dänemark ein und eine minimale EU, begrenzt auf Handelspolitik, Umweltpolitik und technische Zusammenarbeit. ${ }^{44}$ Die Einheitsliste spricht sich gegen eine Mitgliedschaft aus, sieht die EU als „Projekt der großen Geldmaschinerie, in welchem der Profit über den Wohlstand, die Umwelt und Arbeitsbedingungen steht“ und als „Werkzeug für die neoliberale Offensive“45, „undemokratisch und militaristisch“46. Im Folketing sind

37 Vgl. Folketing, Venstre. Danmark og EU, 2010, http://www.ft.dk/Demokrati/Partier/partiernesPolitik/detMenerPartierneOm/EU/Danmark\%20og\%20EU.aspx?party=V (Abruf am 22. September 2011).

38 (Jo før, des bedre) Folketing, Det Radikale Venstre. Danmark og EU, 2009, http://www.ft.dk/ Demokrati/Partier/partiernesPolitik/detMenerPartierneOm/EU/Danmark\%20og\%20EU. aspx?party=B (Abruf am 22. September 2011).

39 Vgl. Peter Yding Brunbech, Centrum-Demokraterne 1973-2008, Aarhus 2010, S. 1, http://danmarkshistorien.dk/leksikon-og-kilder/pdf/materiale/centrumdemokraterne-1973-2008/?pdf=1\& cHash=1393ee77a625e708293317f2b5e46a16 (Abruf am 22. September 2011).

40 Folketing, Socialistik Folkeparti. Danmark og EU, 2009, http://www.ft.dk/Demokrati/Partier/ partiernesPolitik/detMenerPartierneOm/EU/Danmark\%20og\%20EU.aspx?party=F (Abruf am 22. September 2011).

41 Folketing, Det Konservative Folkeparti. Danmark og EU, 2009, http://www.ft.dk/Demokrati/ Partier/partiernesPolitik/detMenerPartierneOm/EU/Danmark\%20og\%20EU.aspx?party=C (Abruf am 22. September 2011).

42 Vgl. Kristendemokraterne, Principprogram 2008, http://www.kd.dk/det-mener-kd/artikler/laesmere/?tx_ttnews\%5Btt_news\%5D=41\&cHash=413920092460d37e6de7cadb0f4e5e38 (Abruf am 22. September 2011).

43 Eigene Übersetzung von: „vi bekæmper alle forsøg på at skabe en europæisk forbundsstat“, Folketing, Dansk Folkeparti. Danmark og EU, 2010, http://www.ft.dk/Demokrati/Partier/partiernesPolitik/detMenerPartierneOm/EU/Danmark\%20og\%20EU.aspx?party=O (Abruf am 22. September 2011).

44 Vgl. Dansk Folkeparti, Danmark og EU. Brochure om Europapolitik og EU-politik, S. 1, http:// www.danskfolkeparti.dk/pictures_org/eu-brochure(1).pdf (Abruf am 6. Mai 2010).

45 Folketing, Enhedslisten - De Rød-Grønne (EL), 2009, S. 3, http://www.ft.dk/ /media/Pdf_materiale/Pdf_publikationer/Informationsark/Partier/enhedslisten\%20pdf.ashx (Abruf am 6. Mai 2010).

46 Enhdeslisten, The Danish Red-Green Alliance, 2006, http://www.enhedslisten.dk/Red-GreenAlliance (Abruf am 7. Mai 2010). 
somit sowohl nicht-euroskeptische als auch euroskeptische Parteien vertreten, die sich unterschiedlich auf die Wahlbündnisse verteilen.

Im Untersuchungszeitraum der 63. bis 66. Wahlperiode schlossen sich die Fraktionen Socialdemokraterne, Socialistisk Folkeparti, Centrum-Demokraterne, Det Radikale Venstre, Enhedslisten nach Wahlen zum linken Wahlbündnis (Venstre) zusammen, während sich im rechten Wahlbündnis (Højre) die Venstre, Dansk Folkeparti, Det Konservative Folkeparti, Liberal Alliance, Fremskridtspartiet und die Kristeligt Folkeparti zusammenschlossen. In der 63. Wahlperiode verfügte das linke Wahlbündnis noch über die Mehrheit (Venstre: 97 - Højre: 81) was zu einer Regierung aus Socialdemokraterne und Radikale Venstre führte. In den darauffolgenden Wahlperioden konnten jedoch die Parteien des rechten Wahlbündnisses mehr Stimmen auf sich vereinen (64. WP: Venstre: 80 - Højre: 99; 65. WP: Venstre: 84 - Højre: 95; 66. WP: Venstre: 82 - Højre: 93). ${ }^{47}$

$\mathrm{Ob}$ die Zugehörigkeit zu einem Wahlbündnis oder der Grad an Euroskeptizismus als erklärender Faktor dient, wurde mit dem Zusammenhangsmaß nach Pearson ( $\mathrm{n}=1.832$ ) berechnet.

\begin{tabular}{|c|c|c|c|c|}
\hline & & $\begin{array}{c}\text { Abstimmungs- } \\
\text { verhalten }\end{array}$ & Wahlbündnis & $\begin{array}{c}\text { Euro- } \\
\text { skeptizismus }\end{array}$ \\
\hline $\begin{array}{l}\text { Abstimmungs- } \\
\text { verhalten }\end{array}$ & $\begin{array}{l}\text { Pearson-Korrelationskoeffizient } \\
\text { Sig. (2-tailed) } \\
\text { N }\end{array}$ & $\begin{array}{r}1 \\
1.832\end{array}$ & $\begin{array}{r}, 04 \\
, 07 \\
1.832\end{array}$ & $\begin{array}{r}, 24^{* *} \\
, 00 \\
1.832\end{array}$ \\
\hline
\end{tabular}

Die Werte der Korrelationskoeffizienten zeigen, dass ein positiver linearer Zusammenhang zwischen den Variablen „Abstimmungsverhalten“ und „Euroskeptizismus“ besteht. Die Wahlbündnisse können demnach nicht als erklärender Faktor einbezogen werden. Die Fraktionen orientieren ihr Abstimmungsverhalten eher an ihrer Grundhaltung zur Europäischen Union und stimmen nicht kohärent innerhalb eines Wahlbündnisses ab.

Auch die Unterscheidung zwischen euroskeptischen und EU-freundlichen Parteien kann nicht als hinreichender Indikator angesehen werden. Das Abstimmungsverhalten zeigt, wie die Anzahl der Ablehnungen steigt, je weiter man sich von den EU-freundlichen Parteien zu den EU-skeptischen bewegt. Die in ihrer Eigendarstellung EU-freundlichen Parteien Venstre, Socialdemokraterne, Det Radikale Venstre und Centrum-Demokraterne lehnen die Positionen der Regierung in sehr seltenen Fällen ab.

Für die 63. Wahlperiode, in der 171 Verhandlungsmandate analysiert wurden, stimmte die Venstre in sieben Fällen (4,1 Prozent) gegen das vorgelegte Mandat, Det konservative Folkeparti, obwohl euroskeptischer, nur sechsmal (3,5 Prozent).

In den 858 Fällen der 64. bis 66. Wahlperiode lehnten die Socialdemokraterne 58 (6,8 Prozent) und die Radikale Venstre 46 (5,6 Prozent) der vorgelegten Verhandlungsmandate

47 Vgl. Rikke Friis Touborg, a.a.O. (Fn. 26). Schwankungen innerhalb der Summen hängen damit zusammen, dass sich in einigen Legislaturperioden grönländische Parteien und Parteien der Färöer-Inseln in den Wahlbündnisse befanden. 


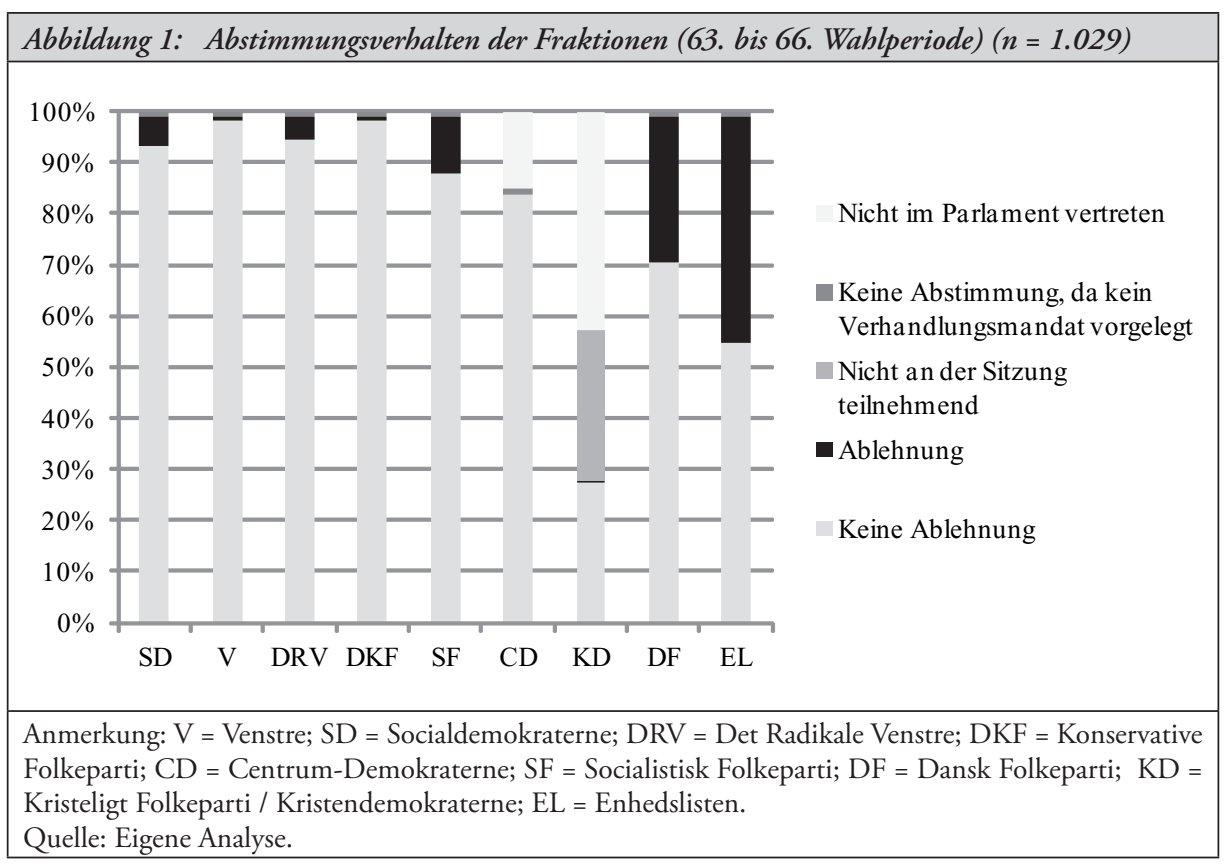

ab. Nie stimmte eine der Regierungsparteien gegen das Verhandlungsmandat des Ministers.

Bei den Parteien aus der Gruppe „Weicher Euroskeptizismus“, Socialistisk Folkeparti und Kristendemokraterne, zeigt sich ein gemischtes Bild. Die Kristendemokraterne stimmten in 282 der 595 Fälle, die in die Zeit ihrer Präsenz im Folketing fielen, für das Verhandlungsmandat und nur fünfmal dagegen. ${ }^{48}$ Die Socialistiske Folkeparti lehnte in 11,0 Prozent der Fälle (113) das präsentierte Mandat ab.

Die Einheitslisten und die Dansk Folkeparti votierten deutlich häufiger gegen Verhandlungsmandate als die anderen Parteien im Folketing. Während sich die Dansk Folkeparti gegen mehr als ein Viertel aller Verhandlungsmandate aussprach (28,6 Prozent), stimmte die EL beinahe gegen die Hälfte aller vorgestellten Mandate (44,6 Prozent).

48 Der Fall der Kristendemokraterne ist jedoch schwer zu interpretieren, da ihre Abgeordneten in den übrigen 302 Fällen der Sitzung fern blieben und in den Beschlussprotokollen die Notiz vermerkt war, dass kein Mitglied der Partei im Ausschuss anwesend war. Eigene Übersetzung: „Kristeligt Folkeparti havde ingen medlemmer i udvalget", siehe beispielsweise Beschlussreferat vom 14. Februar 2003, http://www.euo.dk/dokumenter/ft/euu/arkiv/bilag/2002_2003/ 20030644/ (Abruf am 16. September 2011). Dies war eine Übereinkunft zwischen den Christdemokraten und dem Ausschussvorsitzenden und lag daran, dass die Partei durch das Verlassen von Abgeordneten zu einer anderen Partei nicht mehr die Personalkraft aufbringen konnte, die Sitzungen zu besuchen. Sie hatten aber weiterhin ihren Platz im Ausschuss. Die Abstimmungsregeln besagen, dass eine Partei, die nicht zu den Sitzungen erscheint, obwohl sie einen Sitz hat, automatisch als „nicht-ablehnend“ gezählt wird und somit letzten Endes zustimmt. Dies wollten die Abgeordneten vermeiden und konnten durch ein Übereinkommen mit dem Vorsitzenden erreichen, dass sie als „neutral“ in den Abstimmungen angesehen wurden. 


\subsection{Mandatsänderungen während der Sitzung}

Sollte der Minister sein Mandat während der Sitzungen ändern, würde er damit kurzfristig auf die Wünsche der Parteien reagieren, die ihm ansonsten mit einer Ablehnung drohen können. Jedoch zeigte die Analyse der Protokolle, dass von Oktober 2006 bis Dezember 2009 nur fünfmal das Mandat während der Sitzung geändert wurde.

\begin{tabular}{|l|c|c|c|}
\hline Tabelle 3: Änderungen der Verhandlungsmandate während der Sitzung \\
\hline & Häufigkeit & Prozent & Kumulierte Prozent \\
\hline Nein & 250 & 89,6 & 89,6 \\
Ja & 5 & 1,8 & 91,4 \\
Vertraulich & 16 & 5,7 & 97,1 \\
Kein Verhandlungsmandat zur Debatte & 8 & 2,9 & 100 \\
Total & 279 & 100 & \\
\hline Quelle: Eigene Berechnung.
\end{tabular}

Um Rückschlüsse ziehen zu können, wie oft die Fraktionen während einer Sitzung ihr Fragerecht nutzten, wurden die Nachfragerunden ebenfalls in die Analyse mit einbezogen. In allen 255 Fällen, in denen das Verhandlungsmandat weder zurückgezogen wurde noch der Inhalt vertraulich war, machten die Regierungsparteien erwartungsgemäß am seltensten von ihrem Recht Gebrauch, sich zu äußern. Während sich die Abgeordneten der Venstre in 205 Fällen nicht äußerten, blieben die Parlamentarier der Konservativen Volkspartei sogar in 244 Fällen ohne Beitrag in den Nachfragerunden, und in den übrigen elf Fällen äußerten sie sich nur je einmal.

Die nächste Gruppe bilden die Abgeordneten der Sozialistischen Volkspartei und der Radikalen Venstre. Sie enthielten sich in 43,1 beziehungsweise 42,0 Prozent der Fälle. In 90 Prozent stellten die beiden Parteien drei Nachfragen oder weniger.

Die kritischsten Parteien waren die Einheitsliste, die Dänische Volkspartei und die Sozialdemokraten: Sie nutzten am häufigsten ihr Nachfragerecht. Während die DF nur in 35,7 Prozent aller Fälle keine Stellungnahme abgab, waren es bei der EL 33,7 und bei der SD sogar nur 29,7 Prozent in denen überhaupt nichts zum Verhandlungsmandat gesagt wurde. Hier muss jedoch beachtet werden, dass die Nachfragen in alle Richtungen gehen können, seien es Zustimmungsbekundungen oder Ablehnungen.

Ausgiebige Diskussionen und grundlegende Veränderungen finden demnach nicht statt. $\mathrm{Zu}$ klären ist noch, wie es sich mit der Bearbeitung einer Angelegenheit im Vorfeld der beschlussfassenden Sitzung verhält. Die Interviews mit Abgeordneten des Folketings ergaben, dass im Europaausschuss eine spezielle Situation herrscht, denn anders als in allen anderen Ausschüssen formen hier die europapolitischen „yes-parties“ eine Allianz. Diese Fraktionen sind für die gegenwärtige Regierung die Socialdemokraterne, die Radikale Venstre und die Socialistisk Folkeparti:

„And that is of course a little bit strange, because we [Socialdemokraterne und Regierungsparteien] are not working together in any other political relation in the Folketing. But when it comes to European politics, we work together in most of the cases." ${ }^{49}$

49 Tim Mickler, Interview mit Benny Engelbrecht (Socialdemokraterne) vom 21. Mai 2010 in Kopenhagen. 


\begin{tabular}{|c|c|c|c|c|c|c|c|}
\hline \multicolumn{8}{|c|}{ Tabelle 4: Äußerungen der Fraktionen in den Nachfragerunden } \\
\hline $\begin{array}{l}\text { Anzahl der } \\
\text { Äußerungen }\end{array}$ & V & SD & $\mathrm{DF}$ & DRV & DKF & SF & EL \\
\hline 0 & 205 & 75 & 91 & 107 & 244 & 110 & 86 \\
\hline 1 & 39 & 84 & 97 & 75 & 11 & 55 & 75 \\
\hline 2 & 7 & 49 & 43 & 34 & 0 & 45 & 54 \\
\hline 3 & 3 & 19 & 18 & 20 & 0 & 18 & 31 \\
\hline 4 & 1 & 12 & 3 & 7 & 0 & 14 & 8 \\
\hline 5 & 0 & 12 & 1 & 3 & 0 & 10 & 1 \\
\hline 6 & 0 & 2 & 2 & 6 & 0 & 3 & 0 \\
\hline 7 & 0 & 1 & 0 & 2 & 0 & 0 & 0 \\
\hline 8 & 0 & 1 & 0 & 1 & 0 & 0 & 0 \\
\hline 9 & 0 & 0 & 0 & 0 & 0 & 0 & 0 \\
\hline $\mathrm{n}$ & 255 & 255 & 255 & 255 & 255 & 255 & 255 \\
\hline \multicolumn{8}{|c|}{$\begin{array}{l}\text { Anmerkung: } \mathrm{V}=\text { Venstre; } \mathrm{SD}=\text { Socialdemokraterne; } \mathrm{DRV}=\text { Det Radikale Venstre; DKF = Konservative } \\
\text { Folkeparti; } \mathrm{SF}=\text { Socialistisk Folkeparti; } \mathrm{DF}=\text { Dansk Folkeparti; } \mathrm{EL}=\text { Enhedslisten. } \\
\text { Quelle: Eigene Auszählung. }\end{array}$} \\
\hline
\end{tabular}

Diese Form der Zusammenarbeit hat sich über Jahre etabliert, so Engelbrecht, egal ob es eine „blaue” (Venstre) oder „rote“ (Socialdemokraterne) Regierung gab. In den letzten Jahren zeichnet sich jedoch eine neue Entwicklung ab, in der es für eine Regierung aus dem rechten Wahlbündnis neue Möglichkeiten gibt, sich eine Mehrheit der Stimmen zu sichern, ohne auf die „alten“ Allianzen zurückzugreifen, nämlich mit der Dänischen Volkspartei und der Formung einer „knappen“ Mehrheit:

„But that has changed, as the government is using Danske Folkeparti more and more to form a small, very narrow majority in some cases. "50

Allerdings kann die Regierung in vielen Angelegenheiten nicht auf die Zustimmung der Volkspartei bauen und die Zusammenarbeit mit den EU-freundlichen Parteien auf lange Sicht nicht vollständig durch die neuen Bündnisse ersetzen. Aber die Möglichkeit besteht dennoch und stärkt die Position der Regierung. Informelle Kontakte zwischen den Ministern beziehungsweise Ministerialbeamten und den Parteien sind die Regel, wobei auch hier die EU-Sprecher eine zentrale Rolle einnehmen:

„That is done by bilateral negotiations. So typically we have a situation, where a minister knocks on my door or one of the officials from the ministry that will negotiate the specific matter, in which I am the spokesperson and we take this from there and will negotiate the mandate on bilateral basis. That's pretty normal. “51

Auch Pia Adelsteen (DF) schilderte ein solches Bild für die wenigen Fälle, in denen die Regierung mit der Dansk Folkeparti eine Mehrheit bilden möchte:

50 Ebenda.

51 Ebenda. Dass die Beamten oder Minister die Abgeordneten regelmäßg auch in deren Büros aufsuchen, wird ermöglicht, da das Folketing mit seinem Sitz im Schloss Christiansborg auf Slotsholmen liegt, einer 19 Hektar großen Insel in der Innenstadt Kopenhagens, auf der sich neben vielen Ministerien auch die Büros der Minister und des Premierministers befinden. 
„Because I have ministers who are calling me and inviting me to an informal meeting where we talk and discuss and where I say 'well, then we want this and this'. [...] But actually it is not very often that they are calling me because they know that they are getting the support from other parties." 52

Laut Aussage des Mitarbeiters des EU-Sekretariats wurde diese Entwicklung auch durch die Öffnung der Sitzungen und Protokolle für die Öffentlichkeit unterstützt. ${ }^{53}$ Er betonte, dass es keine Garantie für einen Minister gibt; die Stimmung im Ausschuss könne auch während der Nachfragerunden noch „kippen“ und zu einer Mehrheit der Stimmen gegen die vorgestellte Position führen. Innerhalb dieses Systems werden falsche Äußerungen im Vorfeld der Sitzung des Ministerrates als schwerwiegende Überschreitung eingestuft. Der Europaausschuss nutzt diese Situationen, um seine Stärke zu demonstrieren. So schilderte Pia Adelsteen einen Fall, in dem der Finanzminister unwissentlich eine falsche Aussage im Ausschuss machte und, als dies bekannt wurde, vor den Ausschuss trat, sich in aller Form entschuldigte und infolgedessen strukturelle Veränderungen im Ministerium vorgenommen wurden. ${ }^{54}$

\section{Fazit: Kontrolle und Einfluss außerhalb des Ausschusses}

Der Europaausschuss des dänischen Folketing ist ein sehr anpassungsfähiges Gremium und verfügt formal über ausgeprägte Informations- und Kontrollrechte gegenüber der Regierung. Da die Regierung kaum Mehrheiten gegen die eigene Position vorfand, stellte sich die Frage, ob der Ausschuss das Instrument der Verhandlungsmandate tatsächlich so kraftvoll nutzt, wie gemeinhin angenommen wird. Es musste daher geklärt werden, welche Parteien für die Mehrheit sorgen und ob Einfluss ausgeübt wird.

Die Wahlbündnisse können nicht als Erklärungsmuster dienen, da sie aus EU-freundlichen und EU-skeptischen Parteien bestehen und sich die Parteien in Europafragen eher an ihrer Ausrichtung zur EU als an politischen Verbündeten orientieren. Dies konnte durch die statistische Analyse belegt werden und wurde auch in den Interviews mit den Abgeordneten bestätigt.

Selten findet eine Änderung des Verhandlungsmandats während einer Ausschusssitzung statt. Die Parteien verhalten sich dort wenig konfrontativ, lange Diskussionen um ein Verhandlungsmandat sind eher die Ausnahme. Auch Nachfragen während der Sitzungen sind selten, und es gibt kein Anzeichen dafür, dass die Anzahl der Nachfragerunden zu einer solchen Debatte führen, dass der Minister an seinem Mandat kleinere Korrekturen vornimmt. Insofern deutet vieles darauf hin, dass die Entscheidungen schon vor den Sitzungen gefallen sind.

Die Gespräche mit Abgeordneten und einem Mitarbeiter des EU-Sekretariats kamen zu dem Ergebnis, dass es in der Tat die Abstimmungen im Vorfeld der beschlussfassenden Sitzung sind, die die größte Rolle im parlamentarischen Betrieb spielen. Paradoxerweise hat gerade die Veröffentlichung der Sitzungsprotokolle diese Entwicklung unterstützt, also ein

52 Ders., Interview mit Pia Adelsteen (Dansk Folkeparti) vom 26. Mai 2010 in Kopenhagen.

53 Ders., Interview mit Mitarbeiter des EU-Sekretariats des Folketing vom 27. Mai 2010 in Kopenhagen.

54 Ders., a.a.O. (Fn. 53). 
Akt, der die Transparenz erhöhen sollte. Was sich bereits durch die Analyse der Beschlussprotokolle angedeutet hat, wurde in den Interviews bestätigt. Nicht die Fraktionen aus den Wahlbündnissen, sondern die Oppositionsparteien arbeiten trotz ihrer Stellung mit der Regierung zusammen und nutzen ihre ähnlichen Ansichten, um eine sichere Mehrheit zu formen. Es ist insofern ein glücklicher Umstand, dass gerade die großen Parteien sich hier nicht an der Konfliktlinie „Mehr oder weniger Integration“ orientieren, sondern in diesem - und bemerkenswerterweise auch nur in diesem - Politikfeld kooperieren. Obgleich die dänische Bevölkerung ein gespaltenes Verhältnis zum Integrationsprozess hat, wie man durch die abgelehnten Referenden sehen kann, wird dies durch die Parteien in nationalen Wahlen kaum reflektiert. Dieser Umstand ermöglicht es der Regierung, ein Verhandlungsmandat für den Ministerrat vorzulegen, mit dem sie sich in europäischen Angelegenheiten konstruktiv äußern kann. Dennoch ist der Europaausschuss alles andere als ein „stumpfes Schwert“, sondern muss von der Regierung geachtet werden. Es ist bis ins Jahr 2010 noch nicht vorgekommen, dass sich ein dänischer Minister von seinem Mandat während einer Sitzung des Ministerrates entfernt. Ein Verlassen des Verhandlungsspielraums ist faktisch nicht möglich. „There is no bending the rules there" 55 , wie Pia Adelsteen, EU-Sprecherin der Dansk Folkeparti, es ausdrückte.

Die Fraktionen im Europaausschuss demonstrieren Stärke, sobald es vorkommt, dass ein Minister sich in den Augen der Abgeordneten falsch verhält. Die Minderheitsregierungen sind hier ein wichtiger Faktor, der den Einfluss des Europaausschusses sichert. Die Kontrolle wird immer vor der Androhung eines möglichen Misstrauensvotums ausgeübt. Die Möglichkeit, nicht nur durch einen motivierten Antrag zur Geschäftsordnung die komplette Regierung quasi jederzeit abzuwählen, sondern auch einzelne Minister durch selektive Misstrauensvoten abzusetzen, gibt dem Folketing das nötige Rüstzeug.

Die Fraktionen im Ausschuss stehen in der Tradition eines begleitenden Ausschusses. Schon der Vorläufer des heutigen Ausschusses sollte die Regierung bei den Verhandlungen auf europäischer Ebene mitwirkend begleiten. Diese Sichtweise wird bis heute fortgeführt und auch die EU-freundlichen Oppositionsparteien versuchen eher konstruktiv, ihren Einfluss geltend zu machen, als der amtierenden Regierung durch eine Blockadehaltung die Verhandlungen auf europäischer Ebene zu erschweren, wenngleich der Regierung und der Ministerialbürokratie daher bewusst ist, dass detailliert und kontinuierlich kontrolliert wird. Falschaussagen werden als schwere Vergehen eingestuft, um bereits in einer frühen Phase deutlich zu machen, dass sich die Fraktionen als gewissenhafte Kontrolleure verstehen. Die Möglichkeit der Sanktionierung mit weitreichenden Konsequenzen für die gesamte Regierung ${ }^{56}$ oder einzelne Minister (selektives Misstrauensvotum) veranlasst auch die Ministerialbürokratie nicht, ihren jeweiligen Ressortchef in eine solche Situation zu bringen. Damit ist der Europaausschuss des Folketing in einer starken Position und muss von der Regierung mehr als nur ernst genommen werden.

55 Ebenda.

56 Misstrauensvoten können zu jeder Zeit im Plenum durch einen „motivierten Geschäftsordnungsantrag" (Forslag til motiveret dagsorden) eingereicht und mit einfacher Mehrheit angenommen werden. 